\title{
Features of vascular regulation of students - future specialists in physical education and sports of different sports specializations with different body lengths
}

\author{
Cretu M. ${ }^{1}$, Borysenko I.V. ${ }^{2}$, Ushmarova V.V. ${ }^{3}$, Grynyova V.M. ${ }^{3}$, Masych V.V. ${ }^{4}$
}

\author{
${ }^{1}$ Faculty of Science, Physical Education and Informatics, University of Pitesti, Romania \\ ${ }^{2}$ Department of Olympic and Professional Sports, Sports Games and Tourism, H.S. Skovoroda \\ Kharkiv National Pedagogical University, Kharkiv, Ukraine \\ ${ }^{3}$ Department of Primary and Professional Education Department, H.S. Skovoroda Kharkiv National \\ Pedagogical University, Kharkiv, Ukraine \\ ${ }^{4}$ Department of Physics and Cyber Technical Systems, H.S. Skovoroda Kharkiv National \\ Pedagogical University, Kharkiv, Ukraine
}

\section{DOI: https://doi.org/10.34142/HSR.2021.07.02.03}

\section{How to Cite}

Cretu M., Borysenko I.V., Ushmarova V.V., Grynyova V.M., Masych V.V. Features of vascular regulation of students future specialists in physical education and sports of different sports specializations with different body lengths. Health, Sport, Rehabilitation. 2021;7(2):29-44. https://doi.org/10.34142/HSR.2021.07.02.03

\begin{abstract}
Purpose: to reveal the features of the indicators of the orthostatic test in students with different body lengths and different sport's specializations, studying in the specialty "Physical education and sports".

Material and methods. The study involved 42 students who play sports at the amateur level. The following research methods were used in the work: method of analysis of literary sources; method of determining body length; orthostatic test method; method of determining stroke volume and minute blood volume.

Results. The influence of both factors (body length and sport) on the orthostatic test was significant for the following data: systolic blood pressure in the vertical position, diastolic blood pressure in the vertical position; change in diastolic blood pressure when changing body position from horizontal to vertical; Heart rate in vertical and horizontal positions; change in heart rate during the transition from horizontal to vertical position; stroke volume of blood in the vertical position; change in the stroke volume of blood during the transition from horizontal to vertical position; all indicators of minute volume of a blood-groove $(p<0,005 ; p<0,01 ; p<0,001)$. The more significant influence of judo and football classes in comparison with running short and medium distances on the indicators of vegetative-vascular regulation was determined: the best indicators - in judo, the next place - in football, then - athletes. It was found that students with a body length of more than $190 \mathrm{~cm}$ have difficulty with vegetative-vascular regulation.

Conclusions. To improve the adaptive capacity of vascular regulation to change the position of the body from horizontal to vertical in tall athletes is effective to use any exercise, but the most effective exercises that activate aerobic and anaerobic glycolytic energy systems. Also useful are exercises that require frequent transitions from lying down (sitting) to standing position, as well as changes in the direction of movement.

Key words: orthostatic test, body length, athletes, students
\end{abstract}

(C) Cretu M., Borysenko I.V., Ushmarova V.V., Grynyova V.M., Masych V.V., 2021 


\section{Анотація}

Крету М., Борисенко І.В., Ушмарова В.В., Гриньова В.М., Масіч В.В. Особливості вегетосудинної регуляції студентів - майбутніх фахівців з фізичного виховання і спорту різних спортивних спеціалізацій з різною довжиною тіла

Мета: виявити особливості показників ортостатичної проби у студентів з різною довжиною тіла і різних спортивних спеціалізацій, які навчаються за спеціальністю «Фізичне виховання і спорт».

Матеріал і методи. В дослідженні взяли участь 42 студента - майбутніх фахівців з фізичного виховання і спорту. В роботі застосовувались такі методи дослідження: метод аналізу літературних джерел; метод визначення довжини тіла; метод ортостатичної проби; метод розрахунку ударного об'єму крові та хвилинного об'єму крові. Результати. Вплив обох факторів (довжина тіла і вид спорту) на показники ортостатичної проби виявився достовірним для наступних даних: систолічний артеріальний тиск у вертикальному положенні, діастолічний артеріальний тиск у вертикальному положенні; зміна діастолічного артеріального тиску при зміні положення тіла з горизонтального на вертикальне; ЧСС у вертикальному та горизонтальному положеннях; зміна ЧСС при переході з горизонтального у вертикальне положення; ударний обсяг крові у вертикальному положенні; зміна ударного обсягу крові при переході з горизонтального у вертикальне положення; все показники хвилинного обсягу кровотоку ( $<0,005 ; p<0,01 ; p<0,001)$. Визначено більш суттєвий вплив занять дзюдо та футболом у порівнянні з заняттями бігом на короткі і середні дистанції на показники вегетосудинної регуляції: найліпші показники - у представників дзюдо, наступне місце - у представників футболу, потім - легкоатлети. Виявлено, що у студентів з довжиною тіла понад 190 см спостерігається утруднення вегетосудинної регуляції.

Висновки. Для поліпшення адаптаційних можливостей вегетосудинної регуляції до зміни положення тіла 3 горизонтального на вертикальне у високих спортсменів ефективним є застосування будь-яких фізичних вправ, але найбільш ефективні вправи, які активізують аеробні та анаеробні гліколітичні системи енергозабезпечення. Також корисними є вправи, які вимагають частих переходів з положень лежачи (сидячи) в положення стоячи, а також зміни напрямку рухів.

Ключові слова: ортостатичний тест, довжина тіла, спортсмени, студенти

\section{Аннотация}

Крету М., Борисенко И.В., Ушмарова В.В., Гринева В.Н., Масич В.В. Особенности вегетососудистой регуляции студентов - будущих специалистов по физическому воспитанию и спорту различных спортивных специализаций с разной длиной тела

Цель: выявить особенности показателей ортостатической пробы у студентов с разной длиной тела и разных спортивных специализаций, обучающихся по специальности «Физическое воспитание и спорт».

Материал и методы. В исследовании приняли участие 42 студента - будущих специалистов по физическому воспитанию и спорту. В работе использованы следующие методы исследования: метод анализа литературных источников; метод определения длины тела; метод ортостатической пробы; метод расчёта ударного объема и минутного объема крови.

Результаты. Влияние обоих факторов (длина тела и вид спорта) на показатели ортостатической пробы оказался достоверным для следующих данных: систолическое артериальное давление в вертикальном положении, диастолическое артериальное давление в вертикальном положении; изменение диастолического артериального давления при изменении положения тела из горизонтального в вертикальное чСС в вертикальном и горизонтальном положениях; изменение ЧСС при переходе из горизонтального в вертикальное положение; ударный объем крови в вертикальном положении; изменение ударного объема крови при переходе из горизонтального в вертикальное положение; все показатели минутного объема кровотока ( $p<0,005 ; p<0,01$; $\mathrm{p}<0,001)$. Определены более существенное влияние занятий дзюдо и футболом по сравнению с занятиями бегом на короткие и средние дистанции на показатели вегетососудистой регуляции: лучшие показатели - у представителей дзюдо, следующее место - у представителей футболу, потом - легкоатлеты. Выявлено, что у студентов с длиной тела более 190 см наблюдается затруднение вегетососудистой регуляции.

Выводы. Для улучшения адаптационных возможностей вегетососудистой регуляции к изменению положения тела с горизонтального на вертикальное в высоких спортсменов эффективно применение любых физических упражнений, но наиболее эффективные упражнения, которые активизируют аэробные и анаэробные гликолитические системы энергообеспечения. Также полезны упражнения, которые требуют частых переходов из положений лежа (сидя) в положение стоя, а также изменения направления движения.

Ключевые слова: ортостатическая проба, длина тела, спортсмены, студенты 


\section{Introduction}

Vegetovascular regulation is one of the main mechanisms for ensuring the normal functioning of the body [1-3]. One of the manifestations of vegetative-vascular regulation is vasoconstriction and dilation in response to external influences $[4,5]$. One of the simplest and most accessible methods for determining the quality of vegetative-vascular regulation is the orthostatic test $[6,7]$. The orthostatic test is based on determining the body's adaptation to a change in body position from horizontal to vertical [5-8].

In athletes, the orthostatic test is based on training-induced changes in the functioning of the autonomic nervous system [9-10]. The results of the orthostatic test are influenced by a combination of such external factors as psychological stress, sleep quality, latent diseases, changes in environmental parameters (temperature, altitude), and others [710]. The results of the orthostatic test help to optimize the training process and prevent fatigue in athletes $[2,10,11]$.

Orthostatic test is performed on the basis of measurements of heart rate (HR) and blood pressure $[4,5]$. Changes in heart rate and blood pressure reflect changes in the state of the autonomic nervous system and cardiovascular system. During this test, the indicators of heart rate and blood pressure are measured in the supine position, heart rate in the standing position. The indicators measured during the orthostatic test are a reliable criterion for the load on the autonomic nervous system.

Nazarenko [11] assessed the balance function in athletes and people who do not go in for sports before and after an active orthostatic test. The athletes showed a higher level of balance function in comparison with the control, which decreased to a much lesser extent after an active orthostatic test, which indicates a positive effect of sports on the stability of the statokinetic system. At the same time, statistically significant differences in the balance function between wrestlers and football players appear after an active orthostatic test. In studies [12] it was revealed that athletes - basketball players of taller stature adapt worse to orthostatic load in comparison with athletes of average and below average height.

It is also known [12-14] that in people of high body length, the change in performance during the transition from horizontal to vertical body position is more pronounced, because a larger volume of blood falls sharply to the lower extremities. This leads to the need for more pronounced adaptation mechanisms of heart rate and blood pressure. In our previous study [13], the increase in heart rate in tall people did not differ significantly from this figure in students of medium and below average body length. But blood pressure, both systolic and diastolic, is significantly higher in tall students compared to others. This fact can be regarded as a more pronounced adaptive response of students with a body length above $190 \mathrm{~cm}$ from the cardiovascular system and vascular regulation. These adaptive responses are not sufficient, because the magnitude of the shock volume of blood and minute volume of blood flow in the vertical position in students of high body length is significantly smaller compared to students with a body length of 150-175 $\mathrm{cm}[13,14]$.

Iordanskaya, Buchina [10] revealed the features of orthostatic stability in the vegetative support of the organism's working capacity of highly qualified athletes. The authors examined a group of 203 people, of whom 109 were men and 94 were women in two sports, volleyball (104 athletes) and rowing (99 athletes). The authors have developed a program and criteria for assessing the operational diagnosis of the functional state of the autonomic nervous system, including an orthostatic test with an assessment of heart rate, blood pressure, electrocardiogram in terms of monitoring training and pre-competition loads of the annual training cycle. The interrelation of the level of orthostatic stability in the vegetative support of the working capacity of volleyball players was revealed, taking into account the growth indicators and the playing role. In tall volleyball players of diagonal and central blockers, symptoms of dysadaptation of the cardiovascular system in response to orthostasis are more often diagnosed. In the group of rowing athletes, the relationship between the level of orthostatic stability with age and gender was revealed: young men and juniors more often show symptoms of dysadaptation in response to orthostasis and extreme physical load on the CONCEPT rowing ergometer. It was revealed that the speed-power loads of male rowers are more often reflected in the appearance of hypertension and hypertensive reaction in the work "to failure" on the rowing ergometer. Prompt diagnosis of orthostatic autonomic stability reveals early symptoms of cardiovascular overstrain in a timely manner and serves as a signal for stress correction and recovery.

We $[13,14]$ found that students with a body length of more than $190 \mathrm{~cm}$ have difficulty with vegetative-vascular regulation. The effect of body length on the orthostatic test was also significant for 
the following data: systolic blood pressure in the vertical position, diastolic blood pressure in the vertical position; change in diastolic blood pressure when changing body position from horizontal to vertical; heart rate in vertical and horizontal positions; change in heart rate during the transition from horizontal to vertical position; stroke volume of blood in the vertical position; change in stroke volume of blood during the transition from horizontal to vertical position; all indicators of minute blood volume. However, the question of the joint influence of body length and sports specialization on the indicators of the orthostatic test has not been investigated. There was a significant positive relationship between body length and heart rate $\mathrm{c}$ in horizontal, vertical positions and changes in heart rate when changing body position from horizontal to vertical $(r=0.50-0.71)$. There was also a positive significant relationship between body length and the value of systolic pressure in the vertical position $(r=$ 0.72); negative significant relationship between body length and the value of the stroke volume of blood in the vertical position $(r=-0.65)$.

However, despite the presence of studies showing the effect of body length on the indicators of the orthostatic test in athletes from different sports, this issue remains open and requires additional research. Among athletes, a special place is occupied by students involved in various sports and studying in the specialty "Physical Education and Sports". This category of students is especially significant, since they not only improve their sports skills, but also must bring knowledge to their students in the future.

Purpose: to reveal the features of the indicators of the orthostatic test in students with different body lengths and different sport's specializations, studying in the specialty "Physical education and sports".

\section{Material and methods}

\section{Participants}

The study involved 42 students who play sports at the amateur level. The number of students with a body length of more than $190 \mathrm{~cm}$ was 12 people. The number of students whose body length was $150-175 \mathrm{~cm}$ was 30 people. There were no students with a body length of $176-189 \mathrm{~cm}$ in the study. This was the basis for the division of students into such groups according to body length, because it is known that large values of body length (over 190 $\mathrm{cm})$ negatively affect the adaptation of the cardiovascular system in the transition from horizontal to vertical position.

The study was conducted on the basis of H.S. Skovoroda Kharkiv National Pedagogical University.

\section{Procedure}

The following research methods were used in the work: method of analysis of literature sources; method of determining body length; orthostatic test method; method of determining stroke volume and minute blood volume; methods of statistical data processing (comparison of averages by the Student's method, multivariate analysis of variance and correlation analysis).

An orthostatic test measures heart rate and blood pressure. These values are measured in a horizontal position, then repeat these measurements in the subject after actively getting up in a vertical position at the 10th minute [15-18].

A natural reaction to the orthostatic test is an increase in heart rate. As a result, the minute volume of blood is slightly reduced. In well-trained athletes, heart rate is relatively small and ranges from 5 to 15 beats per minute. In young athletes, the reaction may be more pronounced. Systolic blood pressure either remains unchanged or even decreases slightly (by 2$6 \mathrm{~mm} . \mathrm{Hg}$ ); diastolic blood pressure naturally increases by $10-15 \%$ relative to its value in the horizontal position. If during a 10-minute study the systolic pressure approaches the initial values, the diastolic pressure remains elevated. Signs of orthostatic instability are a sharp drop in blood pressure and a very large increase in heart rate $[9$, 12].

Measurements of body length are performed in a standing position using a vertical height meter. The person stands on a wooden plane with his back to the vertical bar, touching her heels, buttocks, interscapular area with the shoulders set back (head not resting). The arms should be lowered along the torso, the abdomen - tightened, the heels - together, the socks - separately. The position of the head should be such that the upper edge of the earlobe and the lower edge of the orbit are in the same horizontal plane. The movable bar is attached to the head without pressure, but tightly.

The magnitude of the stroke volume depends on the force of the heart contraction and the amount of blood flowing to it during diastole through the veins. Stroke volume (SV) can be calculated by Starr's formula [12-14].

The minute blood volume (MBV) is determined by the stroke volume and heart rate, depending on the position of the human body, its sex, 
age, fitness, environmental conditions and many other factors.

Calculate the minute blood volume (MBV) according to the formula [12]:

$\mathrm{MBV}=\mathrm{SV} \times$ heart rate.

\section{Statistical analysis}

For each indicator, the arithmetic mean, standard deviation $\mathrm{S}$ (standard deviation), error of the mean (m) and estimation of the probability of discrepancies in the Student's t-test with the appropriate level of probability (p) were determined for groups of students involved in football, track and field, judo and for groups of students with body length above average (more than $190 \mathrm{~cm}$ ) and average (below average) $(150-175 \mathrm{~cm})$. Differences and relationships were considered reliable at the significance level $\mathrm{p}<0.05[13,14]$.

Multivariate analysis of variance was also used using a general linear model. The dependent values were heart rate, systolic and diastolic blood pressure, stroke volume and minute blood flow in horizontal and vertical positions, as well as the difference between these indicators in different body

positions. Independent values were body length and sport. Body length of $150-175 \mathrm{~cm}$ was denoted by the number 1, body length greater than $190 \mathrm{~cm}$ was denoted by the number 2 . There were no students with body length values of $175-190 \mathrm{~cm}$ in the study.

We also marked the conditional numbers of the sports that students were involved in: athletics 1; football - 2; judo wrestling - 3) [13, 14].

For statistical processing of the obtained data were used computer programs Microsoft Excel "Data Analysis" - 2013, SPSS - 17.

\section{Results}

Comparison of orthostatic tests of students representatives of different sports showed that the lowest values of heart rate in the horizontal position and in the vertical position of the body in judo. The same applies to blood pressure in the horizontal and vertical position ( $\mathrm{p}<0.001)$ (Table 1-3). The highest heart rate and blood pressure in students - athletes. According to these indicators, footballers occupy an intermediate place.

Table 1

Comparative characteristics of orthostatic test indicators of students involved in track and field and football

\begin{tabular}{|c|c|c|c|c|c|c|c|}
\hline \multirow{2}{*}{ Indicators } & \multirow{2}{*}{ Kind of sport } & \multicolumn{6}{|c|}{ Statistical Indicators } \\
\hline & & $\mathrm{N}$ & $\bar{x}$ & $\mathrm{~S}$ & M & $\mathrm{t}$ & $\mathrm{p}$ \\
\hline \multirow{2}{*}{$\begin{array}{l}\text { Systolic pressure }(\mathrm{mm} . \mathrm{Hg}) \\
\text { in the horizontal position }\end{array}$} & $\begin{array}{l}\text { track and } \\
\text { field }\end{array}$ & 18 & 115.67 & 3.40 & 0.80 & \multirow{2}{*}{5.15} & \multirow{2}{*}{0.000} \\
\hline & football & 12 & 110.00 & 2.09 & 0.60 & & \\
\hline \multirow{2}{*}{$\begin{array}{l}\text { Systolic pressure }(\mathrm{mm} . \mathrm{Hg}) \\
\text { in the vertical position }\end{array}$} & $\begin{array}{l}\text { track and } \\
\text { field }\end{array}$ & 18 & 130.67 & 0.97 & 0.23 & \multirow[t]{2}{*}{2.13} & \multirow[t]{2}{*}{0.043} \\
\hline & football & 12 & 129.00 & 3.13 & 0.90 & & \\
\hline \multirow{2}{*}{$\begin{array}{l}\text { Systolic pressure }(\mathrm{mmHg}) \\
\text { Difference }\end{array}$} & $\begin{array}{l}\text { track and } \\
\text { field }\end{array}$ & 18 & 15.00 & 4.20 & 0.99 & \multirow[t]{2}{*}{-3.22} & \multirow[t]{2}{*}{0.003} \\
\hline & football & 12 & 19.00 & 1.04 & 0.30 & & \\
\hline \multirow{2}{*}{$\begin{array}{l}\text { Diastolic pressure } \\
\text { (mm.Hg.) in the horizontal } \\
\text { position }\end{array}$} & $\begin{array}{c}\text { track and } \\
\text { field }\end{array}$ & 18 & 77.33 & 5.90 & 1.39 & \multirow[t]{2}{*}{1.48} & \multirow[t]{2}{*}{0.150} \\
\hline & football & 12 & 74.00 & 6.27 & 1.81 & & \\
\hline \multirow{2}{*}{$\begin{array}{l}\text { Diastolic pressure } \\
(\mathrm{mm} . \mathrm{Hg} .) \text { in the vertical } \\
\text { position }\end{array}$} & $\begin{array}{l}\text { track and } \\
\text { field }\end{array}$ & 18 & 88.33 & 3.40 & 0.80 & \multirow[t]{2}{*}{9.19} & \multirow[t]{2}{*}{0.000} \\
\hline & football & 12 & 79.00 & 1.04 & 0.30 & & \\
\hline \multirow{2}{*}{$\begin{array}{l}\text { Diastolic pressure } \\
\text { Difference }(\mathrm{mm} . \mathrm{Hg})\end{array}$} & $\begin{array}{l}\text { track and } \\
\text { field }\end{array}$ & 18 & 11.00 & 8.27 & 1.95 & \multirow[t]{2}{*}{2.23} & \multirow[t]{2}{*}{0.034} \\
\hline & football & 12 & 5.00 & 5.22 & 1.51 & & \\
\hline \multirow{2}{*}{$\begin{array}{l}\text { Heart rate (beats. } \mathrm{min}^{-1} \text { ) in } \\
\text { the vertical position }\end{array}$} & $\begin{array}{l}\text { track and } \\
\text { field }\end{array}$ & 18 & 69.33 & 2.11 & 0.50 & \multirow[t]{2}{*}{3.79} & \multirow[t]{2}{*}{0.001} \\
\hline & football & 12 & 58.00 & 12.53 & 3.62 & & \\
\hline \multirow{2}{*}{$\begin{array}{l}\text { Heart rate (beats. } \mathrm{min}^{-1} \text { ) in } \\
\text { the vertical position }\end{array}$} & $\begin{array}{l}\text { track and } \\
\text { field }\end{array}$ & 18 & 71.33 & 5.59 & 1.32 & \multirow[t]{2}{*}{0.65} & \multirow[t]{2}{*}{0.522} \\
\hline & football & 12 & 69.50 & 9.92 & 2.86 & & \\
\hline $\begin{array}{l}\text { Heart rate (beats } \cdot \mathrm{min}^{-1} \text { ) } \\
\text { difference }\end{array}$ & $\begin{array}{l}\text { track and } \\
\text { field }\end{array}$ & 18 & 5.33 & 0.49 & 0.11 & -9.85 & 0.000 \\
\hline
\end{tabular}




\begin{tabular}{|c|c|c|c|c|c|c|c|}
\hline & football & 12 & 11.50 & 2.61 & 0.75 & & \\
\hline \multirow{2}{*}{$\begin{array}{l}\text { Stroke volume }(\mathrm{ml}) \text { in a } \\
\text { horizontal position }\end{array}$} & $\begin{array}{l}\text { track and } \\
\text { field }\end{array}$ & 18 & 60.27 & 8.79 & 2.07 & \multirow[t]{2}{*}{-0.46} & \multirow[t]{2}{*}{0.649} \\
\hline & football & 12 & 61.60 & 5.85 & 1.69 & & \\
\hline \multirow{2}{*}{$\begin{array}{l}\text { Stroke volume }(\mathrm{ml}) \text { in the } \\
\text { vertical position }\end{array}$} & $\begin{array}{l}\text { track and } \\
\text { field }\end{array}$ & 18 & 56.17 & 4.19 & 0.99 & \multirow[t]{2}{*}{-7.73} & \multirow[t]{2}{*}{0.000} \\
\hline & football & 12 & 65.60 & 0.42 & 0.12 & & \\
\hline \multirow{2}{*}{$\begin{array}{l}\text { Stroke volume }(\mathrm{ml}) \\
\text { difference }\end{array}$} & $\begin{array}{l}\text { track and } \\
\text { field }\end{array}$ & 18 & 11.77 & 3.37 & 0.79 & \multirow[t]{2}{*}{4.18} & \multirow[t]{2}{*}{0.000} \\
\hline & football & 12 & 6.00 & 4.18 & 1.21 & & \\
\hline \multirow{2}{*}{$\begin{array}{l}\text { Minute blood volume } \\
\left(1 \cdot \mathrm{min}^{-1}\right) \text { in a horizontal } \\
\text { position }\end{array}$} & $\begin{array}{l}\text { track and } \\
\text { field }\end{array}$ & 18 & 4.17 & 0.55 & 0.13 & \multirow[t]{2}{*}{1.74} & \multirow[t]{2}{*}{0.093} \\
\hline & football & 12 & 3.64 & 1.11 & 0.32 & & \\
\hline \multirow{2}{*}{$\begin{array}{l}\text { Minute blood volume } \\
\left(1 \cdot \mathrm{min}^{-1}\right) \text { in the vertical } \\
\text { position }\end{array}$} & $\begin{array}{l}\text { track and } \\
\text { field }\end{array}$ & 18 & 3.98 & 0.03 & 0.01 & \multirow[t]{2}{*}{-3.92} & \multirow[t]{2}{*}{0.001} \\
\hline & football & 12 & 4.56 & 0.62 & 0.18 & & \\
\hline \multirow{2}{*}{$\begin{array}{l}\text { Minute blood volume } \\
\text { Difference }\left(1 \cdot \mathrm{min}^{-1}\right)\end{array}$} & $\begin{array}{l}\text { track and } \\
\text { field }\end{array}$ & 18 & 0.51 & 0.18 & 0.04 & \multirow[t]{2}{*}{-3.23} & \multirow[t]{2}{*}{0.003} \\
\hline & football & 12 & 0.92 & 0.49 & 0.14 & & \\
\hline \multirow[t]{2}{*}{ Body Length } & $\begin{array}{l}\text { track and } \\
\text { field }\end{array}$ & 18 & 178.33 & 12.24 & 2.89 & \multirow[t]{2}{*}{-0.15} & \multirow[t]{2}{*}{0.882} \\
\hline & football & 12 & 179.00 & 11.49 & 3.32 & & \\
\hline
\end{tabular}

That is, the adaptive capabilities of the cardiovascular system and vegetative-vascular regulation when changing the position of the body from horizontal to vertical in our study were found in students who are engaged in judo. This somewhat contradicts the literature on the best adaptive capabilities of long- and medium-distance running. In our study, athletes were representatives of short and medium distance running. It was found that their adaptive capacity when changing body position is significantly lower compared to football and judo.

Comparative characteristics of orthostatic test indicators of students engaged in track and field and judo

\begin{tabular}{|c|c|c|c|c|c|c|c|}
\hline \multirow{2}{*}{ Indicators } & \multirow{2}{*}{$\begin{array}{l}\text { Kind of } \\
\text { sport }\end{array}$} & \multicolumn{6}{|c|}{ Statistical Indicators } \\
\hline & & $\mathrm{N}$ & $\bar{x}$ & $S$ & $\mathrm{M}$ & $\mathrm{t}$ & $\mathrm{p}$ \\
\hline \multirow{2}{*}{$\begin{array}{l}\text { Systolic pressure } \\
(\mathrm{mm} . \mathrm{Hg}) \text { in the } \\
\text { horizontal position }\end{array}$} & $\begin{array}{l}\text { track and } \\
\text { field }\end{array}$ & 18 & 115.67 & 3.40 & 0.80 & \multirow[t]{2}{*}{6.80} & \multirow[t]{2}{*}{0.000} \\
\hline & judo & 12 & 105.00 & 5.22 & 1.51 & & \\
\hline \multirow{2}{*}{$\begin{array}{l}\text { Systolic pressure } \\
(\mathrm{mm} . \mathrm{Hg}) \text { in the vertical } \\
\text { position }\end{array}$} & $\begin{array}{l}\text { track and } \\
\text { field }\end{array}$ & 18 & 130.67 & 0.97 & 0.23 & \multirow[t]{2}{*}{73.36} & \multirow[t]{2}{*}{0.000} \\
\hline & judo & 12 & 110.00 & 0.00 & 0.00 & & \\
\hline \multirow{2}{*}{$\begin{array}{l}\text { Systolic pressure } \\
\text { (mmHg) } \\
\text { difference }\end{array}$} & $\begin{array}{l}\text { track and } \\
\text { field }\end{array}$ & 18 & 15.00 & 4.20 & 0.99 & \multirow[t]{2}{*}{5.80} & \multirow[t]{2}{*}{0.000} \\
\hline & judo & 12 & 5.00 & 5.22 & 1.51 & & \\
\hline \multirow{2}{*}{$\begin{array}{l}\text { Diastolic pressure } \\
(\mathrm{mm} . \mathrm{Hg} .) \text { in the } \\
\text { horizontal position }\end{array}$} & $\begin{array}{l}\text { track and } \\
\text { field }\end{array}$ & 18 & 77.33 & 5.90 & 1.39 & \multirow[t]{2}{*}{3.34} & \multirow[t]{2}{*}{0.002} \\
\hline & Judo & 12 & 66.00 & 12.53 & 3.62 & & \\
\hline \multirow{2}{*}{$\begin{array}{l}\text { Diastolic pressure } \\
(\mathrm{mm} . \mathrm{Hg} .) \text { in the } \\
\text { vertical position }\end{array}$} & $\begin{array}{c}\text { track and } \\
\text { field }\end{array}$ & 18 & 88.33 & 3.40 & 0.80 & \multirow[t]{2}{*}{22.97} & \multirow[t]{2}{*}{0.000} \\
\hline & Judo & 12 & 65.00 & 1.04 & 0.30 & & \\
\hline \multirow{2}{*}{$\begin{array}{l}\text { Diastolic pressure } \\
\text { Difference }(\mathrm{mm} . \mathrm{Hg})\end{array}$} & $\begin{array}{l}\text { track and } \\
\text { field }\end{array}$ & 18 & 11.00 & 8.27 & 1.95 & \multirow[t]{2}{*}{-0.83} & \multirow[t]{2}{*}{0.415} \\
\hline & Judo & 12 & 13.00 & 1.04 & 0.30 & & \\
\hline \multirow{2}{*}{$\begin{array}{l}\text { Heart rate (beats. } \min ^{-1} \text { ) } \\
\text { in the vertical position }\end{array}$} & $\begin{array}{c}\text { track and } \\
\text { field }\end{array}$ & 18 & 69.33 & 2.11 & 0.50 & \multirow[t]{2}{*}{29.15} & \multirow[t]{2}{*}{0.000} \\
\hline & Judo & 12 & 48.50 & 1.57 & 0.45 & & \\
\hline
\end{tabular}




\begin{tabular}{|c|c|c|c|c|c|c|c|}
\hline \multirow{2}{*}{$\begin{array}{l}\text { Heart rate (beats } \cdot \min ^{-1} \text { ) } \\
\text { in the vertical position }\end{array}$} & $\begin{array}{l}\text { track and } \\
\text { field }\end{array}$ & 18 & 71.33 & 5.59 & 1.32 & \multirow[t]{2}{*}{7.26} & \multirow[t]{2}{*}{0.000} \\
\hline & Judo & 12 & 59.50 & 0.52 & 0.15 & & \\
\hline \multirow{2}{*}{$\begin{array}{l}\text { Heart rate (beats } \cdot \mathrm{min}^{-1} \text { ) } \\
\text { difference }\end{array}$} & $\begin{array}{l}\text { track and } \\
\text { field }\end{array}$ & 18 & 5.33 & 0.49 & 0.11 & \multirow[t]{2}{*}{-20.12} & \multirow[t]{2}{*}{0.000} \\
\hline & Judo & 12 & 11.00 & 1.04 & 0.30 & & \\
\hline \multirow{2}{*}{$\begin{array}{l}\text { Stroke volume }(\mathrm{ml}) \text { in a } \\
\text { horizontal position }\end{array}$} & $\begin{array}{l}\text { track and } \\
\text { field }\end{array}$ & 18 & 60.27 & 8.79 & 2.07 & \multirow[t]{2}{*}{-1.66} & \multirow[t]{2}{*}{0.108} \\
\hline & Judo & 12 & 67.90 & 16.40 & 4.73 & & \\
\hline \multirow{2}{*}{$\begin{array}{l}\text { Stroke volume }(\mathrm{ml}) \text { in } \\
\text { the vertical position }\end{array}$} & $\begin{array}{l}\text { track and } \\
\text { field }\end{array}$ & 18 & 56.17 & 4.19 & 0.99 & \multirow[t]{2}{*}{-12.31} & \multirow[t]{2}{*}{0.000} \\
\hline & Judo & 12 & 71.50 & 1.15 & 0.33 & & \\
\hline \multirow{2}{*}{$\begin{array}{l}\text { Stroke volume (ml) } \\
\text { difference }\end{array}$} & $\begin{array}{c}\text { track and } \\
\text { field }\end{array}$ & 18 & 11.77 & 3.37 & 0.79 & \multirow[t]{2}{*}{-3.83} & \multirow[t]{2}{*}{0.001} \\
\hline & Judo & 12 & 16.80 & 3.76 & 1.09 & & \\
\hline \multirow{2}{*}{$\begin{array}{l}\text { Minute blood volume } \\
\left(1 \cdot \mathrm{min}^{-1}\right) \text { in a horizontal } \\
\text { position }\end{array}$} & $\begin{array}{l}\text { track and } \\
\text { field }\end{array}$ & 18 & 4.17 & 0.55 & 0.13 & \multirow[t]{2}{*}{3.22} & \multirow[t]{2}{*}{0.003} \\
\hline & Judo & 12 & 3.32 & 0.90 & 0.26 & & \\
\hline \multirow{2}{*}{$\begin{array}{l}\text { Minute blood volume } \\
\left(1 \cdot \mathrm{min}^{-1}\right) \text { in the vertical } \\
\text { position }\end{array}$} & $\begin{array}{l}\text { track and } \\
\text { field }\end{array}$ & 18 & 3.98 & 0.03 & 0.01 & \multirow[t]{2}{*}{-22.13} & \multirow[t]{2}{*}{0.000} \\
\hline & Judo & 12 & 4.25 & 0.03 & 0.01 & & \\
\hline \multirow{2}{*}{$\begin{array}{l}\text { Minute blood volume } \\
\text { Difference }\left(1 \cdot \mathrm{min}^{-1}\right)\end{array}$} & $\begin{array}{c}\text { track and } \\
\text { field }\end{array}$ & 18 & 0.51 & 0.18 & 0.04 & \multirow[t]{2}{*}{-1.91} & \multirow[t]{2}{*}{0.067} \\
\hline & Judo & 12 & 0.94 & 0.93 & 0.27 & & \\
\hline \multirow[t]{2}{*}{ Body Length } & $\begin{array}{c}\text { track and } \\
\text { field }\end{array}$ & 18 & 178.33 & 12.24 & 2.89 & \multirow[t]{2}{*}{5.43} & \multirow[t]{2}{*}{0.000} \\
\hline & Judo & 12 & 159.00 & 1.04 & 0.30 & & \\
\hline
\end{tabular}

We can explain the results by the fact that both football and judo are a load of mixed aerobicanaerobic orientation, while running short and medium distances is mainly a job that requires creatine-phosphate and glycolytic mechanisms of energy supply.

Table 3

Comparative characteristics of orthostatic test indicators of students involved in football and judo

\begin{tabular}{|c|c|c|c|c|c|c|c|}
\hline \multirow{2}{*}{ Indikators } & \multirow{2}{*}{$\begin{array}{c}\text { Kind of } \\
\text { sport }\end{array}$} & \multicolumn{6}{|c|}{ Statistical Indikators } \\
\hline & & $\mathrm{N}$ & $\bar{x}$ & $\mathrm{~S}$ & $\mathrm{~m}$ & $\mathrm{t}$ & $p$ \\
\hline \multirow{2}{*}{$\begin{array}{l}\text { Systolic pressure } \\
(\mathrm{mm} . \mathrm{Hg}) \text { in the } \\
\text { horizontal position }\end{array}$} & Football & 12 & 110.00 & 2.09 & 0.60 & \multirow[b]{2}{*}{3.08} & \multirow[b]{2}{*}{0.005} \\
\hline & Judo & 12 & 105.00 & 5.22 & 1.51 & & \\
\hline \multirow{2}{*}{$\begin{array}{l}\text { Systolic pressure } \\
(\mathrm{mm} . \mathrm{Hg}) \text { in the } \\
\text { vertical position }\end{array}$} & football & 12 & 129.00 & 3.13 & 0.90 & \multirow[b]{2}{*}{21.01} & \multirow[b]{2}{*}{0.000} \\
\hline & Judo & 12 & 110.00 & 0.00 & 0.00 & & \\
\hline \multirow{2}{*}{$\begin{array}{l}\text { Systolic pressure } \\
(\mathrm{mmHg}) \text { difference }\end{array}$} & football & 12 & 19.00 & 1.04 & 0.30 & \multirow{2}{*}{9.11} & \multirow{2}{*}{0.000} \\
\hline & Judo & 12 & 5.00 & 5.22 & 1.51 & & \\
\hline \multirow{2}{*}{$\begin{array}{l}\text { Diastolic pressure } \\
(\mathrm{mm} . \mathrm{Hg} .) \text { in the } \\
\text { horizontal position }\end{array}$} & football & 12 & 74.00 & 6.27 & 1.81 & \multirow[b]{2}{*}{1.98} & \multirow[b]{2}{*}{0.061} \\
\hline & Judo & 12 & 66.00 & 12.53 & 3.62 & & \\
\hline \multirow{2}{*}{$\begin{array}{l}\text { Diastolic pressure } \\
(\mathrm{mm} . \mathrm{Hg} .) \text { in the } \\
\text { vertical position }\end{array}$} & football & 12 & 79.00 & 1.04 & 0.30 & \multirow[b]{2}{*}{32.83} & \multirow[b]{2}{*}{0.000} \\
\hline & Judo & 12 & 65.00 & 1.04 & 0.30 & & \\
\hline \multirow{2}{*}{$\begin{array}{l}\text { Diastolic pressure } \\
\text { Difference }(\mathrm{mm} . \mathrm{Hg})\end{array}$} & football & 12 & 5.00 & 5.22 & 1.51 & \multirow{2}{*}{-5.20} & \multirow{2}{*}{0.000} \\
\hline & Judo & 12 & 13.00 & 1.04 & 0.30 & & \\
\hline \multirow{2}{*}{$\begin{array}{l}\text { Heart rate } \\
\text { (beats· } \min ^{-1} \text { ) in the } \\
\text { vertical position }\end{array}$} & football & 12 & 58.00 & 12.53 & 3.62 & \multirow[b]{2}{*}{2.61} & \multirow[b]{2}{*}{0.016} \\
\hline & judo & 12 & 48.50 & 1.57 & 0.45 & & \\
\hline
\end{tabular}




\begin{tabular}{|c|c|c|c|c|c|c|c|}
\hline \multirow{2}{*}{$\begin{array}{l}\text { Heart rate } \\
\text { (beats· } \min ^{-1} \text { ) in the } \\
\text { vertical position }\end{array}$} & football & 12 & 69.50 & 9.92 & 2.86 & \multirow[b]{2}{*}{3.49} & \multirow[b]{2}{*}{0.002} \\
\hline & judo & 12 & 59.50 & 0.52 & 0.15 & & \\
\hline \multirow{2}{*}{$\begin{array}{l}\text { Heart rate } \\
\left(\text { beats } \cdot \mathrm{min}^{-1}\right) \\
\text { difference }\end{array}$} & football & 12 & 11.50 & 2.61 & 0.75 & \multirow[b]{2}{*}{0.62} & \multirow[b]{2}{*}{0.544} \\
\hline & judo & 12 & 11.00 & 1.04 & 0.30 & & \\
\hline \multirow{2}{*}{$\begin{array}{l}\text { Stroke volume }(\mathrm{ml}) \text { in } \\
\text { a horizontal position }\end{array}$} & football & 12 & 61.60 & 5.85 & 1.69 & \multirow{2}{*}{-1.25} & \multirow{2}{*}{0.223} \\
\hline & judo & 12 & 67.90 & 16.40 & 4.73 & & \\
\hline \multirow{2}{*}{$\begin{array}{l}\text { Stroke volume }(\mathrm{ml}) \text { in } \\
\text { the vertical position }\end{array}$} & football & 12 & 65.60 & 0.42 & 0.12 & \multirow{2}{*}{-16.72} & \multirow{2}{*}{0.000} \\
\hline & judo & 12 & 71.50 & 1.15 & 0.33 & & \\
\hline \multirow{2}{*}{$\begin{array}{l}\text { Stroke volume }(\mathrm{ml}) \\
\text { difference }\end{array}$} & football & 12 & 6.00 & 4.18 & 1.21 & \multirow{2}{*}{-6.66} & \multirow{2}{*}{0.000} \\
\hline & judo & 12 & 16.80 & 3.76 & 1.09 & & \\
\hline \multirow{2}{*}{$\begin{array}{l}\text { Minute blood volume } \\
\left(1 \cdot \mathrm{min}^{-1}\right) \text { in a } \\
\text { horizontal position }\end{array}$} & football & 12 & 3.64 & 1.11 & 0.32 & \multirow[b]{2}{*}{0.78} & \multirow[b]{2}{*}{0.442} \\
\hline & judo & 12 & 3.32 & 0.90 & 0.26 & & \\
\hline \multirow{2}{*}{$\begin{array}{l}\text { Minute blood volume } \\
\left(1 \cdot \mathrm{min}^{-1}\right) \text { in the } \\
\text { vertical position }\end{array}$} & football & 12 & 4.56 & 0.62 & 0.18 & \multirow[b]{2}{*}{1.68} & \multirow[b]{2}{*}{0.107} \\
\hline & judo & 12 & 4.25 & 0.03 & 0.01 & & \\
\hline \multirow{2}{*}{$\begin{array}{l}\text { Minute blood volume } \\
\text { Difference }\left(1 \cdot \mathrm{min}^{-1}\right)\end{array}$} & football & 12 & 0.92 & 0.49 & 0.14 & \multirow{2}{*}{-0.07} & \multirow{2}{*}{0.944} \\
\hline & judo & 12 & 0.94 & 0.93 & 0.27 & & \\
\hline \multirow{2}{*}{ Body Length } & football & 12 & 179.00 & 11.49 & 3.32 & \multirow{2}{*}{6.01} & \multirow{2}{*}{0.000} \\
\hline & judo & 12 & 159.00 & 1.04 & 0.30 & & \\
\hline
\end{tabular}

Significant influence of both body length and sport on most indicators of orthostatic test was found (Table 4). The results of analysis of variance confirmed the results of comparing the averages of the Student's t-test. The influence of sport on heart rate, systolic and diastolic pressure, as well as the calculated values of stroke volume and minute blood flow in the supine and standing positions was significant for almost all indicators ( $p<0.05$; $p$ $<0.001$ ) (Table 4). The only exception is the rate of stroke blood volume in the horizontal position ( $p>$ 0.05) (Table 4). Thus, the analysis of variance confirmed the results of comparing the means of the Student's t-test for the best effect on vascular regulation and the state of the cardiovascular system in judo. Short and medium distance running by students at the level of mass discharges has a less pronounced effect on orthostatic test performance. Football classes occupy an intermediate place between judo and athletics in terms of impact on vascular regulation.

Body length also significantly affects the indicators of vascular regulation (Table 4). There is a significant effect of body length on systolic blood pressure in the standing position, diastolic blood pressure in the supine and standing positions, heart rate in the supine and standing positions, stroke blood volume in the standing position, minute blood flow in the supine and standing positions $(\mathrm{p}<0.001$ ) (Table 4).
The influence of both factors (body length and sport) on the orthostatic test was also significant for the following data: systolic blood pressure in the vertical position, diastolic blood pressure in the vertical position; change in diastolic blood pressure when changing body position from horizontal to vertical; Heart rate in vertical and horizontal positions; change in heart rate during the transition from horizontal to vertical position; stroke volume of blood in the vertical position; change in the stroke volume of blood during the transition from horizontal to vertical position; all indicators of minute volume of a blood-groove ( $\mathrm{p}<0.005 ; \mathrm{p}<0.01 ; \mathrm{p}<0.001)$.

The influence of both factors (body length and sport) on the orthostatic test was also significant for the following data: systolic blood pressure in the vertical position, diastolic blood pressure in the vertical position; change in diastolic blood pressure when changing body position from horizontal to vertical; Heart rate in vertical and horizontal positions; change in heart rate during the transition from horizontal to vertical position; stroke volume of blood in the vertical position; change in the stroke volume of blood during the transition from horizontal to vertical position; all indicators of minute volume of a blood-groove ( $\mathrm{p}<0.005$; $\mathrm{p}<0.01 ; \mathrm{p}<0.001)$. 
Indicators of multivariate analysis of variance of the influence of sport and body length on the orthography of students (Tests of Between-Subjects Effects)

\begin{tabular}{|c|c|c|c|c|c|c|}
\hline \multirow[b]{2}{*}{ Source } & \multirow[b]{2}{*}{ Dependent Variable } & \multicolumn{5}{|c|}{ Tests of Between-Subjects Effects } \\
\hline & & $\begin{array}{l}\text { Type III } \\
\text { Sum of } \\
\text { Squares }\end{array}$ & Df & $\begin{array}{l}\text { Mean } \\
\text { Square }\end{array}$ & $\mathrm{F}$ & Sig. \\
\hline \multirow{15}{*}{$\begin{array}{l}\text { Corrected } \\
\text { Model }\end{array}$} & $\begin{array}{l}\text { Systolic pressure }(\mathrm{mm} . \mathrm{Hg}) \text { in the horizontal } \\
\text { position }\end{array}$ & $888.000 \mathrm{a}$ & 4 & 222 & 16.695 & 0.000 \\
\hline & $\begin{array}{l}\text { Systolic pressure }(\mathrm{mm} . \mathrm{Hg}) \text { in the } \\
\text { vertical position }\end{array}$ & $3560.571 b$ & 4 & 890.143 & 2744.607 & 0.000 \\
\hline & $\begin{array}{l}\text { Systolic pressure }(\mathrm{mmHg}) \\
\text { difference }\end{array}$ & $1280.571 c$ & 4 & 320.143 & 19.742 & 0.000 \\
\hline & $\begin{array}{l}\text { Diastolic pressure }(\mathrm{mm} . \mathrm{Hg} .) \text { in the } \\
\text { horizontal position }\end{array}$ & $1373.143 d$ & 4 & 343.286 & 5.484 & 0.001 \\
\hline & $\begin{array}{l}\text { Diastolic pressure }(\mathrm{mm} . \mathrm{Hg} .) \text { in the } \\
\text { vertical position }\end{array}$ & $4053.000 \mathrm{e}$ & 4 & 1013.25 & 430.922 & 0.000 \\
\hline & Diastolic pressure Difference $(\mathrm{mm} . \mathrm{Hg})$ & $806.143 f$ & 4 & 201.536 & 6.81 & 0.000 \\
\hline & $\begin{array}{l}\text { Heart rate }\left(\text { beats } \cdot \mathrm{min}^{-1}\right) \text { in the vertical } \\
\text { position }\end{array}$ & 4994.143g & 4 & 1248.536 & 1184.508 & 0.000 \\
\hline & $\begin{array}{l}\text { Heart rate }\left(\text { beats } \cdot \mathrm{min}^{-1}\right) \text { in the vertical } \\
\text { position }\end{array}$ & $2452.286 \mathrm{~h}$ & 4 & 613.071 & 92.21 & 0.000 \\
\hline & $\begin{array}{l}\text { Heart rate (beats } \cdot \mathrm{min}^{-1} \text { ) } \\
\text { difference }\end{array}$ & $437.571 \mathrm{i}$ & 4 & 109.393 & 269.836 & 0.000 \\
\hline & Stroke volume $(\mathrm{ml})$ in a horizontal position & $824.096 j$ & 4 & 206.024 & 1.786 & 0.152 \\
\hline & Stroke volume $(\mathrm{ml})$ in the vertical position & $1959.673 k$ & 4 & 489.918 & 128.314 & 0.000 \\
\hline & Stroke volume $(\mathrm{ml})$ difference & 1027.7831 & 4 & 256.946 & 44.508 & 0.000 \\
\hline & $\begin{array}{l}\text { Minute blood volume }\left(1 \cdot \mathrm{min}^{-1}\right) \text { in a } \\
\text { horizontal position }\end{array}$ & $19.290 \mathrm{~m}$ & 4 & 4.823 & 12.729 & 0.000 \\
\hline & $\begin{array}{l}\text { Minute blood volume }\left(1 \cdot \mathrm{min}^{-1}\right) \text { in the } \\
\text { vertical position }\end{array}$ & $6.619 n$ & 4 & 1.655 & 2065.344 & 0.000 \\
\hline & Minute blood volume Difference $\left(1 \cdot \mathrm{min}^{-1}\right)$ & 4.7840 & 4 & 1.196 & 4.536 & 0.004 \\
\hline \multirow{12}{*}{ Intercept } & $\begin{array}{l}\text { Systolic pressure }(\mathrm{mm} . \mathrm{Hg}) \text { in the horizontal } \\
\text { position }\end{array}$ & 438703.7 & 1 & 438703.7 & 32991.95 & 0.000 \\
\hline & $\begin{array}{l}\text { Systolic pressure }(\mathrm{mm} . \mathrm{Hg}) \text { in the } \\
\text { vertical position }\end{array}$ & 556475 & 1 & 556475 & 1715798 & 0.000 \\
\hline & $\begin{array}{l}\text { Systolic pressure }(\mathrm{mmHg}) \\
\text { difference }\end{array}$ & 6993.204 & 1 & 6993.204 & 431.248 & 0.000 \\
\hline & $\begin{array}{l}\text { Diastolic pressure (mm.Hg.) in the } \\
\text { horizontal position }\end{array}$ & 194326.9 & 1 & 194326.9 & 3104.531 & 0.000 \\
\hline & $\begin{array}{l}\text { Diastolic pressure }(\mathrm{mm} . \mathrm{Hg} .) \text { in the } \\
\text { vertical position }\end{array}$ & 225666.2 & 1 & 225666.2 & 95972.97 & 0.000 \\
\hline & Diastolic pressure Difference $(\mathrm{mm} . \mathrm{Hg})$ & 3104.92 & 1 & 3104.92 & 104.915 & 0.000 \\
\hline & $\begin{array}{l}\text { Heart rate }\left(\text { beats } \cdot \mathrm{min}^{-1}\right) \text { in the vertical } \\
\text { position }\end{array}$ & 124863.2 & 1 & 124863.2 & 118460 & 0.000 \\
\hline & $\begin{array}{l}\text { Heart rate }\left(\text { beats } \cdot \mathrm{min}^{-1}\right) \text { in the vertical } \\
\text { position }\end{array}$ & 163461 & 1 & 163461 & 24585.6 & 0.000 \\
\hline & $\begin{array}{l}\text { Heart rate (beats } \cdot \mathrm{min}^{-1} \text { ) } \\
\text { difference }\end{array}$ & 3048.124 & 1 & 3048.124 & 7518.705 & 0.000 \\
\hline & Stroke volume $(\mathrm{ml})$ in a horizontal position & 137842.2 & 1 & 137842.2 & 1194.89 & 0.000 \\
\hline & Stroke volume $(\mathrm{ml})$ in the vertical position & 141439 & 1 & 141439 & 37044.27 & 0.000 \\
\hline & Stroke volume $(\mathrm{ml})$ difference & 4050.128 & 1 & 4050.128 & 701.567 & 0.000 \\
\hline
\end{tabular}




\begin{tabular}{|c|c|c|c|c|c|c|}
\hline & $\begin{array}{l}\text { Minute blood volume }\left(1 \cdot \mathrm{min}^{-1}\right) \text { in a } \\
\text { horizontal position }\end{array}$ & 483.904 & 1 & 483.904 & 1277.286 & 0.000 \\
\hline & $\begin{array}{l}\text { Minute blood volume }\left(1 \cdot \mathrm{min}^{-1}\right) \text { in the } \\
\text { vertical position }\end{array}$ & 634.008 & 1 & 634.008 & 791360.4 & 0.000 \\
\hline & Minute blood volume Difference $\left(1 \cdot \mathrm{min}^{-1}\right)$ & 21.647 & 1 & 21.647 & 82.09 & 0.000 \\
\hline \multirow{15}{*}{$\begin{array}{l}\text { Kind of } \\
\text { sport }\end{array}$} & $\begin{array}{l}\text { Systolic pressure }(\mathrm{mm} . \mathrm{Hg}) \text { in the horizontal } \\
\text { position }\end{array}$ & 631.75 & 2 & 315.875 & 23.755 & 0.000 \\
\hline & $\begin{array}{l}\text { Systolic pressure }(\mathrm{mm} . \mathrm{Hg}) \text { in the } \\
\text { vertical position }\end{array}$ & 2467 & 2 & 1233.5 & 3803.292 & 0.000 \\
\hline & $\begin{array}{l}\text { Systolic pressure }(\mathrm{mmHg}) \\
\text { difference }\end{array}$ & 945.75 & 2 & 472.875 & 29.161 & 0.000 \\
\hline & $\begin{array}{l}\text { Diastolic pressure }(\mathrm{mm} . \mathrm{Hg} .) \text { in the } \\
\text { horizontal position }\end{array}$ & 427 & 2 & 213.5 & 3.411 & 0.044 \\
\hline & $\begin{array}{l}\text { Diastolic pressure }(\mathrm{mm} . \mathrm{Hg} .) \text { in the } \\
\text { vertical position }\end{array}$ & 3172 & 2 & 1586 & 674.506 & 0.000 \\
\hline & Diastolic pressure Difference (mm.Hg) & 349 & 2 & 174.5 & 5.896 & 0.006 \\
\hline & $\begin{array}{l}\text { Heart rate (beats } \cdot \mathrm{min}^{-1} \text { ) in the vertical } \\
\text { position }\end{array}$ & 4441.75 & 2 & 2220.875 & 2106.984 & 0.000 \\
\hline & $\begin{array}{l}\text { Heart rate (beats } \cdot \mathrm{min}^{-1} \text { ) in the vertical } \\
\text { position }\end{array}$ & 1588 & 2 & 794 & 119.423 & 0.000 \\
\hline & $\begin{array}{l}\text { Heart rate }\left(\text { beats } \cdot \mathrm{min}^{-1}\right) \\
\text { difference }\end{array}$ & 418.75 & 2 & 209.375 & 516.458 & 0.000 \\
\hline & Stroke volume $(\mathrm{ml})$ in a horizontal position & 140.507 & 2 & 70.254 & 0.609 & 0.549 \\
\hline & Stroke volume $(\mathrm{ml})$ in the vertical position & 1642.17 & 2 & 821.085 & 215.05 & 0.000 \\
\hline & Stroke volume $(\mathrm{ml})$ difference & 636.077 & 2 & 318.039 & 55.091 & 0.000 \\
\hline & $\begin{array}{l}\text { Minute blood volume }\left(1 \cdot \mathrm{min}^{-1}\right) \text { in a } \\
\text { horizontal position }\end{array}$ & 11.741 & 2 & 5.871 & 15.496 & 0.000 \\
\hline & $\begin{array}{l}\text { Minute blood volume }\left(1 \cdot \mathrm{min}^{-1}\right) \text { in the } \\
\text { vertical position }\end{array}$ & 2.603 & 2 & 1.302 & 1624.679 & 0.000 \\
\hline & Minute blood volume Difference $\left(1 \cdot \mathrm{min}^{-1}\right)$ & 2.996 & 2 & 1.498 & 5.681 & 0.007 \\
\hline \multirow{14}{*}{ Body lengtr } & $\begin{array}{l}\text { Systolic pressure }(\mathrm{mm} . \mathrm{Hg}) \text { in the horizontal } \\
\text { position }\end{array}$ & 15.429 & 1 & 15.429 & 1.16 & 0.288 \\
\hline & $\begin{array}{l}\text { Systolic pressure }(\mathrm{mm} . \mathrm{Hg}) \text { in the } \\
\text { vertical position }\end{array}$ & 42.857 & 1 & 42.857 & 132.143 & 0.000 \\
\hline & $\begin{array}{l}\text { Systolic pressure }(\mathrm{mmHg}) \\
\text { difference }\end{array}$ & 6.857 & 1 & 6.857 & 0.423 & 0.520 \\
\hline & $\begin{array}{l}\text { Diastolic pressure (mm.Hg.) in the } \\
\text { horizontal position }\end{array}$ & 289.714 & 1 & 289.714 & 4.628 & 0.038 \\
\hline & $\begin{array}{l}\text { Diastolic pressure }(\mathrm{mm} . \mathrm{Hg} .) \text { in the } \\
\text { vertical position }\end{array}$ & 96.429 & 1 & 96.429 & 41.01 & 0.000 \\
\hline & Diastolic pressure Difference $(\mathrm{mm} . \mathrm{Hg})$ & 51.857 & 1 & 51.857 & 1.752 & 0.194 \\
\hline & $\begin{array}{l}\text { Heart rate (beats } \cdot \mathrm{min}^{-1} \text { ) in the vertical } \\
\text { position }\end{array}$ & 685.714 & 1 & 685.714 & 650.549 & 0.000 \\
\hline & $\begin{array}{l}\text { Heart rate (beats } \cdot \mathrm{min}^{-1} \text { ) in the vertical } \\
\text { position }\end{array}$ & 189 & 1 & 189 & 28.427 & 0.000 \\
\hline & $\begin{array}{l}\text { Heart rate (beats } \cdot \mathrm{min}^{-1} \text { ) } \\
\text { difference }\end{array}$ & 34.714 & 1 & 34.714 & 85.629 & 0.000 \\
\hline & Stroke volume $(\mathrm{ml})$ in a horizontal position & 248.919 & 1 & 248.919 & 2.158 & 0.150 \\
\hline & Stroke volume $(\mathrm{ml})$ in the vertical position & 56.679 & 1 & 56.679 & 14.845 & 0.000 \\
\hline & Stroke volume $(\mathrm{ml})$ difference & 8.297 & 1 & 8.297 & 1.437 & 0.238 \\
\hline & $\begin{array}{l}\text { Minute blood volume }\left(1 \cdot \mathrm{min}^{-1}\right) \text { in a } \\
\text { horizontal position }\end{array}$ & 6.432 & 1 & 6.432 & 16.977 & 0.000 \\
\hline & $\begin{array}{l}\text { Minute blood volume }\left(1 \cdot \mathrm{min}^{-1}\right) \text { in the } \\
\text { vertical position }\end{array}$ & 2.403 & 1 & 2.403 & 2999.62 & 0.000 \\
\hline
\end{tabular}




\begin{tabular}{|c|c|c|c|c|c|c|}
\hline & Minute blood volume Difference $\left(1 \cdot \mathrm{min}^{-1}\right)$ & 0.693 & 1 & 0.693 & 2.63 & 0.113 \\
\hline \multirow{15}{*}{$\begin{array}{l}\text { Kind of } \\
\text { sport· Body } \\
\text { length }\end{array}$} & $\begin{array}{l}\text { Systolic pressure }(\mathrm{mm} . \mathrm{Hg}) \text { in the horizontal } \\
\text { position }\end{array}$ & 42.857 & 1 & 42.857 & 3.223 & 0.081 \\
\hline & $\begin{array}{l}\text { Systolic pressure }(\mathrm{mm} . \mathrm{Hg}) \text { in the } \\
\text { vertical position }\end{array}$ & 84 & 1 & 84 & 259 & 0.000 \\
\hline & $\begin{array}{l}\text { Systolic pressure }(\mathrm{mmHg}) \\
\text { Difference }\end{array}$ & 6.857 & 1 & 6.857 & 0.423 & 0.520 \\
\hline & $\begin{array}{l}\text { Diastolic pressure ( } \mathrm{mm} . \mathrm{Hg} .) \text { in the } \\
\text { horizontal position }\end{array}$ & 207.429 & 1 & 207.429 & 3.314 & 0.077 \\
\hline & $\begin{array}{l}\text { Diastolic pressure ( } \mathrm{mm} . \mathrm{Hg} \text {.) in the } \\
\text { vertical position }\end{array}$ & 21 & 1 & 21 & 8.931 & 0.005 \\
\hline & Diastolic pressure Difference (mm.Hg) & 360.429 & 1 & 360.429 & 12.179 & 0.001 \\
\hline & $\begin{array}{l}\text { Heart rate (beats } \cdot \mathrm{min}^{-1} \text { ) in the vertical } \\
\text { position }\end{array}$ & 1344 & 1 & 1344 & 1275.077 & 0.000 \\
\hline & $\begin{array}{l}\left.\text { Heart rate (beats } \cdot \mathrm{min}^{-1}\right) \text { in the vertical } \\
\text { position }\end{array}$ & 1296.429 & 1 & 1296.429 & 194.991 & 0.000 \\
\hline & $\begin{array}{l}\text { Heart rate }\left(\text { beats } \cdot \mathrm{min}^{-1}\right) \\
\text { Difference }\end{array}$ & 51.857 & 1 & 51.857 & 127.914 & 0.000 \\
\hline & Stroke volume $(\mathrm{ml})$ in a horizontal position & 183.639 & 1 & 183.639 & 1.592 & 0.215 \\
\hline & Stroke volume $(\mathrm{ml})$ in the vertical position & 92.61 & 1 & 92.61 & 24.255 & 0.000 \\
\hline & Stroke volume (ml) difference & 326.469 & 1 & 326.469 & 56.551 & 0.000 \\
\hline & $\begin{array}{l}\text { Minute blood volume }\left(1 \cdot \mathrm{min}^{-1}\right) \text { in a } \\
\text { horizontal position }\end{array}$ & 9.219 & 1 & 9.219 & 24.334 & 0.000 \\
\hline & $\begin{array}{l}\text { Minute blood volume }\left(1 \cdot \mathrm{min}^{-1}\right) \text { in the } \\
\text { vertical position }\end{array}$ & 2.46 & 1 & 2.46 & 3070.976 & 0.000 \\
\hline & Minute blood volume Difference $\left(1 \cdot \mathrm{min}^{-1}\right)$ & 2.627 & 1 & 2.627 & 9.964 & 0.003 \\
\hline \multirow{15}{*}{ Error } & $\begin{array}{l}\text { Systolic pressure }(\mathrm{mm} . \mathrm{Hg}) \text { in the horizontal } \\
\text { position }\end{array}$ & 492 & 37 & 13.297 & - & - \\
\hline & $\begin{array}{l}\text { Systolic pressure }(\mathrm{mm} . \mathrm{Hg}) \text { in the } \\
\text { vertical position }\end{array}$ & 12 & 37 & 0.324 & - & - \\
\hline & $\begin{array}{l}\text { Systolic pressure }(\mathrm{mmHg}) \\
\text { Difference }\end{array}$ & 600 & 37 & 16.216 & - & - \\
\hline & $\begin{array}{l}\text { Diastolic pressure ( } \mathrm{mm} . \mathrm{Hg} .) \text { in the } \\
\text { horizontal position }\end{array}$ & 2316 & 37 & 62.595 & - & - \\
\hline & $\begin{array}{l}\text { Diastolic pressure }(\mathrm{mm} . \mathrm{Hg} .) \text { in the } \\
\text { vertical position }\end{array}$ & 87 & 37 & 2.351 & - & - \\
\hline & Diastolic pressure Difference $(\mathrm{mm} . \mathrm{Hg})$ & 1095 & 37 & 29.595 & - & - \\
\hline & $\begin{array}{l}\left.\text { Heart rate (beats } \cdot \mathrm{min}^{-1}\right) \text { in the vertical } \\
\text { position }\end{array}$ & 39 & 37 & 1.054 & - & - \\
\hline & $\begin{array}{l}\left.\text { Heart rate (beats } \cdot \mathrm{min}^{-1}\right) \text { in the vertical } \\
\text { position }\end{array}$ & 246 & 37 & 6.649 & - & - \\
\hline & $\begin{array}{l}\text { Heart rate }\left(\text { beats } \cdot \mathrm{min}^{-1} \text { ) }\right. \\
\text { Difference }\end{array}$ & 15 & 37 & 0.405 & - & - \\
\hline & Stroke volume $(\mathrm{ml})$ in a horizontal position & 4268.31 & 37 & 115.36 & - & - \\
\hline & Stroke volume $(\mathrm{ml})$ in the vertical position & 141.27 & 37 & 3.818 & - & - \\
\hline & Stroke volume (ml) difference & 213.6 & 37 & 5.773 & - & - \\
\hline & $\begin{array}{l}\text { Minute blood volume }\left(1 \cdot \mathrm{min}^{-1}\right) \text { in a } \\
\text { horizontal position }\end{array}$ & 14.018 & 37 & 0.379 & - & - \\
\hline & $\begin{array}{l}\text { Minute blood volume }\left(1 \cdot \mathrm{min}^{-1}\right) \text { in the } \\
\text { vertical position }\end{array}$ & 0.03 & 37 & 0.001 & - & - \\
\hline & Minute blood volume Difference $\left(1 \cdot \mathrm{min}^{-1}\right)$ & 9.757 & 37 & 0.264 & - & - \\
\hline \multirow[t]{2}{*}{ Total } & $\begin{array}{l}\text { Systolic pressure }(\mathrm{mm} . \mathrm{Hg}) \text { in the horizontal } \\
\text { position }\end{array}$ & 518862 & 42 & - & - & - \\
\hline & Systolic pressure $(\mathrm{mm} . \mathrm{Hg})$ in the & 652344 & 42 & - & - & - \\
\hline
\end{tabular}


vertical position

Systolic pressure $(\mathrm{mmHg})$

Difference

9294

42

Diastolic pressure (mm.Hg.) in the horizontal position

$228384 \quad 42$

Diastolic pressure (mm.Hg.) in the vertical position

$266262 \quad 42$

Diastolic pressure Difference $(\mathrm{mm} . \mathrm{Hg})$

Heart rate (beats $\cdot \mathrm{min}^{-1}$ ) in the vertical

position

$5982 \quad 42$

Heart rate (beats $\cdot \mathrm{min}^{-1}$ ) in the vertical

position

$156954 \quad 42$

Heart rate (beats $\cdot \mathrm{min}^{-1}$ )

difference

Stroke volume $(\mathrm{ml})$ in a horizontal position

Stroke volume $(\mathrm{ml})$ in the vertical position

Stroke volume $(\mathrm{ml})$ difference

$193656 \quad 42$

Minute blood volume $\left(1 \cdot \mathrm{min}^{-1}\right)$ in a

horizontal position

$3642 \quad 42$

Minute blood volume $\left(1 \cdot \mathrm{min}^{-1}\right)$ in the

vertical position

$170884.4 \quad 42$

$170086.6 \quad 42$

$6851.22 \quad 42$

Minute blood volume Difference $\left(1 \cdot \mathrm{min}^{-1}\right) \quad 38.022$

Systolic pressure $(\mathrm{mm} . \mathrm{Hg})$ in the horizontal position

1380

41

Systolic pressure $(\mathrm{mm} . \mathrm{Hg})$ in the vertical position

$631.834 \quad 42$

Systolic pressure $(\mathrm{mmHg})$

difference

$3572.571 \quad 41$

Diastolic pressure (mm.Hg.) in the

horizontal position

$1880.571 \quad 41$

Diastolic pressure (mm.Hg.) in the

vertical position

Corrected

Diastolic pressure Difference (mm.Hg)

Heart rate (beats $\cdot \mathrm{min}^{-1}$ ) in the vertical

position

$3689.143 \quad 41$

Heart rate (beats $\cdot \mathrm{min}^{-1}$ ) in the vertical

position

Heart rate (beats $\cdot \mathrm{min}^{-1}$ )

difference

$4140 \quad 41$

Stroke volume $(\mathrm{ml})$ in a horizontal position $5092.406 \quad 41$

Stroke volume $(\mathrm{ml})$ in the vertical position $\quad 2100.943 \quad 41$

Stroke volume $(\mathrm{ml})$ difference $\quad 1241.383 \quad 41$

Minute blood volume $\left(1 \cdot \mathrm{min}^{-1}\right)$ in a

horizontal position

$33.308 \quad 41$

Minute blood volume $\left(1 \cdot \mathrm{min}^{-1}\right)$ in the

vertical position

$6.648 \quad 41$

Minute blood volume Difference $\left(1 \cdot \mathrm{min}^{-1}\right) \quad 14.541 \quad 41$

a. $R$ Squared $=0.643$ (Adjusted R Squared = 0.605); b. R Squared =0.997 (Adjusted R Squared =0.996); c. R Squared $=0.681$ (Adjusted R Squared $=0.646$ ); . $R$ Squared $=0.372$ (Adjusted R Squared $=0.304$ ); e. $R$ Squared $=0.979$ (Adjusted R Squared =0.977); f. R Squared $=0.424$ (Adjusted R Squared $=0.362) ;$ g. R Squared $=0.992$ (Adjusted R Squared $=0.991$ ); h. $R$ Squared $=0.909$ (Adjusted R Squared =0.899); i. R Squared $=0.967$ (Adjusted R Squared $=0.963$ ); . $R$ Squared $=0.162$ (Adjusted R Squared =0.071); k. R Squared $=0.933$ (Adjusted R Squared $=0.925$ ); I. R Squared $=0.828$ (Adjusted R Squared $=0.809$ ); $\mathrm{m}$. R Squared $=0.579$ (Adjusted R Squared $=0.534$ ); $\mathrm{n}$. R Squared =0.996 (Adjusted R Squared $=0.995$ ); o. $R$ Squared $=0.329$ (Adjusted $R$ Squared $=0.256$ ) 


\section{Discussion}

The purpose of the work set in this study was fully confirmed. It has been shown that students who engage in different sports have different adaptive capabilities in terms of vascular regulation. It was also shown that students with a body length greater than $190 \mathrm{~cm}$ have less adaptive capacity for vascular regulation compared to students of medium and below average body length.

Our data confirmed the results of other authors $[4,9,10]$, that in athletes orthostatic instability associated with decreased venous tone develops relatively rarely. However, when conducting orthostatic tests, it can sometimes be detected [12]. Therefore, the use of orthostatic tests to assess the functional state of the body of athletes is considered appropriate.

A natural reaction to the orthostatic test is an increase in heart rate $[1,4,6]$. Due to this, the minute volume of blood flow is reduced slightly. In welltrained athletes, heart rate is relatively small and ranges from 5 to 15 beats $\cdot$ min- 1 . In young athletes, the reaction may be more pronounced [9]. Systolic blood pressure either remains unchanged or even decreases slightly (by $2-6 \mathrm{~mm} \mathrm{Hg}$ ); diastolic blood pressure naturally increases by $10-15 \%$ relative to its value in the horizontal position. If during 10 - minute research systolic pressure approaches initial values, arterial pressure remains raised.

Signs of orthostatic instability are a sharp drop in blood pressure and a very large increase in heart rate [4]. But at the present stage, a simple assessment of the orthostatic sample according to heart rate continues to be refined. The fact is that such a seemingly reliable indicator, which is the increase in heart rate in the vertical position relative to the heart rate in the horizontal position, sometimes gives inaccurate data. This is especially true for athletes with bradycardia in a horizontal position: their heart rate can increase by $30-25$ beats $\cdot$ min 1 without any signs of orthostatic instability. In this regard, it is recommended to evaluate the orthostatic test on the basis of the actual heart rate in the vertical position of the body. If the heart rate does not exceed 89 beats per minute for 10 minutes, the reaction is considered normal. A heart rate of $90-95$ beats $\cdot$ min-1 indicates a decrease in orthostatic stability, and a heart rate exceeding 95 beats $\cdot$ min-1 indicates low resistance to changes in body position in space, at which orthostatic collapse is possible. This approach to the assessment of orthostatic reactions is based on the socalled principle of invariance, the essence of which is that under the influence of a perturbing effect, the performance of the body's autonomic systems do not depend (or depend to a small extent) on baseline and are determined exclusively current needs of the organism $[4,12]$.

The response to the orthostatic test improves under the influence of sports training $[10,11]$. And this applies to all athletes, not just those sports in which a change of body position is a mandatory element.

According to the literature $[2,4,8]$, orthostatic hypotension and orthostatic collapse are abnormal, pathological phenomena. Osadchy [4] writes that a decrease in systemic blood pressure is often accompanied by dizziness, blurred vision, sweating and even loss of consciousness with a sudden transition from horizontal (or sitting) to a vertical position. Physiological effects of this change in body position are the result of increased hydrostatic pressure in veins and the arteries of the lower and lowering - in the vessels of the upper half of the body and the corresponding redistribution of blood mass.

Cerebral circulation is protected from fluctuations in hydrostatic pressure in the vascular system due to the same pressure of this factor on both intravascular and extravascular (in the spinal canal) pressure. Under these conditions, the cerebral tie is under the control of systemic blood pressure and therefore a decrease in the latter immediately leads to a decrease in the arteriovenous gradient

Therefore, despite the presence of nervous and humoral mechanisms that reduce the resistance of cerebral vessels and prevent the effects of oscillations of systemic arterial suppression [4], an essential factor in maintaining cerebral blood flow is adequate blood pressure [4].

Thus, in those individuals in whom the orthostatic is written off above the compensatory mechanisms can not prevent a significant decrease in blood pressure, decreased cerebral blood flow, which causes "fainting" or "unconsciousness" (depending on the intensity of the above symptoms of cerebral circulatory disorders). In the scientific literature, "fainting" (or "syncope", "collapse") entered as the most important element of the syndrome, referred to as "orthostatic hypotension". This term refers to such conditions in which the violation of circulatory homeostasis during a change in body position is the main pathogenetic mechanism of the disease, and the main manifestation - low average blood pressure [4].Thus, we obtained results that confirm the results of the literature on the difficult vascular regulation of tall people [12-14]. In addition, these results are clarified by the fact that a significant increase not only in diastolic blood pressure, but also systolic, in 
the transition from horizontal to vertical position. But these changes are insufficient for the adaptation to changes in body position in tall students was similar to students whose body length does not exceed the average.

Based on the results, the following recommendations can be made: to improve the adaptive capacity of vascular regulation to change body position from horizontal to vertical, it is effective to use any exercise, but the most effective exercises that activate aerobic and anaerobic glycolytic energy systems. In addition, exercises that require frequent transitions from lying down (sitting) to standing position, as well as changes in the direction of movement are useful [19,20].

\section{Conclusions}

1. The influence of both factors (body length and sport) on the orthostatic test was significant for the following data: systolic blood pressure in the vertical position, diastolic blood pressure in the vertical position; change in diastolic blood pressure when changing body position from horizontal to vertical; Heart rate in vertical and horizontal positions; change in heart rate during the transition from horizontal to vertical position; stroke volume of blood in the vertical position; change in the stroke volume of blood during the transition from horizontal to vertical position; all indicators of minute volume of a blood-groove $(p<0.005 ; p<0.01 ; p<0.001)$.

2 . The more significant influence of judo and football classes in comparison with running short and medium distances on the indicators of vegetativevascular regulation was determined: the best indicators - in judo, the next place - in football, then - athletes. It was found that students with a body length of more than $190 \mathrm{~cm}$ have difficulty with vegetative-vascular regulation. To improve the adaptive capacity of vascular regulation to change the position of the body from horizontal to vertical in tall athletes is effective to use any exercise, but the most effective exercises that activate aerobic and anaerobic glycolytic energy systems. Also useful are exercises that require frequent transitions from lying down (sitting) to standing position, as well as changes in the direction of movement.

\section{Acknowledgments}

The authors are very grateful to all the athletes and coaches who took part in the experiment.

The study was conducted according to: research work on the topic of the Department of Olympic and Professional Sports, Sports Games and Tourism of H.S. Skovoroda Kharkiv National Pedagogical University for 2021-2026: "Development and substantiation of technologies for health promotion and harmonious development of people of different ages and social groups" (№ state registration: 0121U110053).

\section{Conflict of interest}

The authors declare that there is no conflict of interest.

\section{References}

1. Buhr LK, Stack CI, Luetkemeier MJ. The Effects Of Hydration Status On Pulse Rate And Heart Rate Variability During A Stand Test Of Orthostatic Tolerance. Medicine and Science in Sports and Exercise. 2013;45(5):37-42.

2. Hynynen E, Iglesias X, Feriche B, Calderon C, Abalos $X$, Vazquez J, et al. Heart Rate Variability in Orthostatic Test During Different Training Periods in Elite Swimmers. Medicine and Science in Sports and Exercise. 2012;44:782-787.

3. Kubala P, Smorawinski J, Kaciuba-Uscilko H, Nazar K, Bicz B, Greenleaf JE. Effect of three day bed-rest on circulatory and hormonal responses to active orthostatic test in endurance trained athletes and untrained subjects. Journal of gravitational physiology: a journal of the International Society for Gravitational Physiology. 1996;3(2):40-1.

4. Osadchiy LI. Body position and regulation of blood circulation. Nauka. 1982: 144 p. In Russian
Nazarenko AS. Equilibrium function response in athletes after orthostatic test. Physiological journal. 2016: 25-29. In Russian

5. Rodrigues GD, Goncalves TR, De Souza SC, Da Silva Soares PP. Comparison of Cardiac Vagal Modulation From the Orthostatic Stress Test Between Untrained Individuals and Athletes. Medicine and Science in Sports and Exercise. 2014;46(5):341-2.

6. Rodriguez FA, Iglesias X, Feriche B, Calderon C, Abalos X, Vazquez J, et al. Effects of Altitude Training on Heart Rate Variability in Orthostatic Test in Elite Swimmers. Medicine and Science in Sports and Exercise. 2012;44:364-372.

7. Vesterinen V, Nummela A. Nocturnal Heart Rate Variability and Morning Orthostatic Test as Tools to Monitor Training Load. Medicine and Science in Sports and Exercise. 2018;50(5):118-9.

8. Pavy-Le Traon A, Sigaudo D, Vasseur P, Maillet A, Fortrat JO, Hughson RL, et al. Cardiovascular responses to orthostatic tests after a 42-day head-down bed-rest. European Journal of Applied Physiology. 1998;77(1-2):50-9.

9. Lutfullin II, Al'metova RR. Heart rate variability in 
young hockey players at rest and during active orthostatic test. Fiziologiia cheloveka. 2014;40(2):105-11.

10. Jordanskaya FA, Buchina EV. Orthostatic stability in the vegetative support of the performance of highly qualified athletes. Medical and biological problems of sports. 2017: 26-34. In Russian

11. Nazarenko AS. Equilibrium function response in athletes after orthostatic test. Physiological journal. 2016: 25-29. In Russian

12. Kozina ZL, Slyusarev VF. Unconventional methods of improving the efficiency of basketball players. Physical Education, Sports and Health Culture in Modern Society. 2002; 2: 199-201. In Russian

13. Kozina Z, Borysenko I, Grynyova V, Masych V, Ushmarova V. Influence of sports specialization and body length on orthostatic test indicators of students majoring in "Physical Education and Sports". Journal of Physical Education and Sport. 2021; 21 (3): 1580 1586. https://doi.org/10.7752/jpes.2021.03200

14. Borysenko I, Marian C, Kozina Z. Influence of body length on orthostatic test parameters of studentathletes. Zdorov'â, sport, reabilìtaciâ [Health, sport, rehabilitation]. 2020;6(4):47-57. https://doi.org/10.34142/HSR.2020.06.04.05

15. Schafer D, Olstad BH, Wilhelm M. Can Heart Rate Variability Segment Length During Orthostatic Test Be Reduced To 2 Min? Medicine and Science in Sports and Exercise. 2015;47(5):48-53.

16. Wolthuis RA, Hull DH, Fischer JR, McAfoose DA, Curtis JT. Blood-pressure variability of the individual in orthostatic testing. Aviation Space and
Environmental Medicine. 1979;50(8):774-7.

17. Roberson KB, Signorile J, Singer C, Jacobs K, Eltoukhy M, Ruta N, et al. Hemodynamic Responses to an Exercise Stress Test in Parkinson's Disease Patients without Orthostatic Hypotension. Medicine and Science in Sports and Exercise. 2019;51(6):292297.

18. Murray RH, Bowers JA, Goltra ER. Comparison of footboard and saddle supports for orthostatic tests on a tilt table. Journal of applied physiology. 1966;21(4):1409-11.

19. Iermakov SS, Kozina ZhL, Ceslitska M, Mushketa R, Krzheminski M, Stankevich B. Razrabotka kompyuternyih programm dlya opredeleniya psihofiziologicheskih vozmozhnostey i svoystv nervnoy sistemyi lyudey $\mathrm{s}$ raznyim urovnem fizicheskoy aktivnosti [Computer program development for determination of psychophysiological possibilities and properties of thenervous system of people with the different level of physicalactivity]. Zdorov'â, sport, reabilitaciâ [Health, sport, rehabilitation]. 2016;2(1):14-19.

20. Kozina ZL, Goloborodko YA, Boichuk YD, Sobko IM, Repko OO, Bazilyuk TA, et.al. The influence of a special technique for developing coordination abilities on the level of technical preparedness and development of psycho-physiological functions of young volleyball players 14-16 years of age. Journal of Physical Education and Sport. 2018; 18(3):1445-1454. DOI:10.7752/jpes.2018.03214

\section{Information about authors}

\section{Cretu M.}

https://orcid.org/0000-0003-1934-0534

cmaryan_mc@yahoo.com

Faculty of Science, Physical Education and Informatics; University of Pitesti

Str. Targul din Vale, nr.1, 110040 Pitesti, Arges, Romania

\section{Borvsenko I.V.}

irynaborysenko13@gmail.com

H. S. Skovoroda Kharkiv National Pedagogical University

Alchevskikh st. 29, Kharkiv, 61002, Ukraine

\section{Ushmarova V.V.}

victoria.uschmarova@gmail.com https://orcid.org/0000-0002-9306-5553

H.S. Skovoroda Kharkiv National Pedagogical University Alchevskikh st. 29, Kharkiv, 61002, Ukraine

\section{Grynyova V.M.}

kvn.grineva@gmail.com

http://orcid.org/0000-0002-3027-4622

H.S. Skovoroda Kharkiv National Pedagogical University

Altshevskih str. 29, Kharkiv, 61002, Ukraine

\section{Masych V.V.}

masych@hnpu.edu.ua

http://orcid.org/0000-0002-8943-7756

H.S. Skovoroda Kharkiv National Pedagogical University

Altshevskih str. 29, Kharkiv, 61002, Ukraine 


\section{Інформація про авторів}

\section{Крету М.}

https://orcid.org/0000-0003-1934-0534

cmaryan_mc@yahoo.com

Факультет науки, фізичного виховання та інформатики, Університет Пітесті

вул. Таргундін Вале, 1, 110040, Пітесті, Румунія

\section{Борисенко I.B.}

irynaborysenko13@gmail.com

Харківський національний педагогічний університет імені Г.С. Сковороди

вул. Алчевських, 29, м. Харків, 61002, Україна

\section{Ушмарова В.В.}

victoria.uschmarova@gmail.com

https://orcid.org/0000-0002-9306-5553

Харківський національний педагогічний університет імені Г.С. Сковороди вул. Алчевських, 29, м. Харків, 61002, Україна

\section{Гриньова В.М.}

http://orcid.org/0000-0002-3027-4622

kvn.grineva@gmail.com

Харківський національний педагогічний університет імені Г.С. Сковороди вул. Алчевських 29, Харків, 61002, Україна

\section{Масіч B.B.}

http://orcid.org/0000-0002-8943-7756

masych@hnpu.edu.ua

Харківський національний педагогічний університет імені Г.С. Сковороди

вул. Алчевських 29, Харків, 61002, Україна

\section{Информация об авторах}

\section{Kрету M.}

https://orcid.org/0000-0003-1934-0534

cmaryan_mc@yahoo.com

Факультет науки, физического воспитания и информатики, Университет Питести

Ул. Таргундин Вале, 1, 110040, Питести, Румыния

\section{Борисенко И.В.}

irynaborysenko13@gmail.com

Харьковский национальный педагогический университет имени Г.С. Сковороды ул. Алчевских 29, Харьков, 61002, Украина

\section{Уимарова В.В.}

victoria.uschmarova@gmail.com

https://orcid.org/0000-0002-9306-5553

Харьковский национальный педагогический университет имени Г.С. Сковороды ул. Алчевских 29, Харьков, 61002, Украина

\section{Гринева В.H.}

http://orcid.org/0000-0002-3027-4622

kvn.grineva@gmail.com

Харьковский национальный педагогический университет имени Г.С. Сковороды ул. Алчевских 29, Харьков, 61002, Украина

\section{Масич В.В.}

http://orcid.org/0000-0002-8943-7756

masych@hnpu.edu.ua

Харьковский национальный педагогический университет имени Г.С. Сковороды ул. Алчевских 29, Харьков, 61002, Украина

This work is licensed under a Creative Commons Attribution 4.0 International License (CC BY 4.0)

Received: 2021-04-15 Accepted: 2021-05-10 Published: 2021-06-25 